\title{
Smartfone como alicerce de metodologias ativas no ensino $e$ aprendizagem da geografia
}

As novas tecnologias decompuseram definitivamente as formas, hábitos e costumes do como vivemos, o que de certo é um caminho sem volta. Embora pareça simples, em um primeiro olhar, trilhar esse caminho requer reflexão de todos, porquanto nos exige novas questões éticas e morais, das quais não estávamos acostumados, especialmente no âmbito educacional. Desta forma, este tem como objetivo o levantamento de aplicativos livres disponíveis em lojas virtuais (Aplle Store e Play Store) e sites, bem como, a avaliação como são capazes de auxiliar e quais entraves podem oferecer no processo de ensino e aprendizagem da Geografia. Os alunos podem se beneficiar desses recursos disponibilizados, no que tange a sua produção acadêmica e realizando atividades colaborativas com colegas e professores, estes alunos se beneficiam das tecnologias que são oferecidas em uma série de recursos, que se integrados aos processos pedagógicos, auxiliam e aproximam as escolas da realidade tecnológica, em que a sociedade atual está inserida. Desta forma, observamos que a internet é um dos fatos limitantes na utilização destas novas tecnologias, bem como, o tamanho dos aplicativos disponibilizados, o excesso de propagandas nos aplicativos, além das limitações próprias das suas funções em versões livre.

\section{Smartfone as the foundation of active methodologies in teaching and learning geography}

\begin{abstract}
New technologies have definitely broken down the forms, habits and customs of how we live, which is certainly a path of no return. Although it seems simple, at first glance, following this path requires reflection by everyone, as it demands new ethical and moral questions, which we were not used to, especially in the educational field. Thus, it aims to survey free applications available in virtual stores (Aplle Store and Play Store) and websites, as well as assess how they are able to assist and what barriers they can offer in the process of teaching and learning Geography. Students can benefit from these resources made available, with regard to their academic production and performing collaborative activities with colleagues and teachers, these students benefit from the technologies that are offered in a series of resources, which, if integrated with the pedagogical processes, help and approach the schools of technological reality, in which today's society is inserted. Thus, we observe that the internet is one of the limiting facts in the use of these new technologies, as well as, the size of the applications made available, the excess of advertisements in the applications, in addition to the limitations of their functions in free versions.
\end{abstract}

Keywords: Applications; Site; Geography; Free versions.

Topic: Tecnologia, Educomunicação e Cibercultura

Reviewed anonymously in the process of blind peer
Received: $12 / 03 / 2020$

Approved: $\mathbf{1 0 / 0 5 / 2 0 2 0}$
Edinelson Saldanha Correa (iD)

Universidade Federal do Oeste do Pará, Brasil

http://lattes.cnpq.br/0344768485868201

http://orcid.org/0000-0002-4162-4296

Edinelson_saldanha@hotmail.com

Thiago Rocha Shinaigger (iD

Universidade Federal do Oeste do Pará, Brasil

http://lattes.cnpq.br/6476976978923362

http://orcid.org/0000-0002-2691-6039

shinaigger@gmail.com
Referencing this:

CORREA, E. S.; SHINAIGGER, T. R.. Smartfone como alicerce de metodologias ativas no ensino e aprendizagem da geografia. Educationis, v.8, n.2, p.19-28, 2020. DOI: http://doi.org/10.6008/CBPC2318-3047.2020.002.0003 


\section{INTRODUÇÃO}

Em um mundo cada vez mais conectado, oferecendo uma infinidade de conteúdo, ou ainda, tornando o processo de comunicação mais fácil, isso unido a popularização da internet e dos smartfone, tem tornado mais dificultoso ter a atenção dos estudantes, refletindo, portanto, no processo de ensino e aprendizagem em sala de aula. Isso possibilita que a sociedade contemporânea imponha diversos desafios no âmbito educacional, na qual o maior acesso às informações em seus diversos meios de divulgação, em especial, a internet, potencializam a necessidade de aprimoramento das técnicas de mediação no processo de ensino e aprendizagem. Os discentes são "bombardeados" por informações, todavia, devemos repensar como convertê-las em conhecimento significativo para eles (GOIS, 2018).

Segundo Moran (2015), se queremos que os alunos sejam proativos, precisamos adotar metodologias nas quais os alunos se envolvam em atividades cada vez mais complexas, em que tenham que tomar decisões e avaliar os resultados, com o apoio de materiais relevantes. Se queremos que sejam criativos, eles precisam experimentar inúmeras novas possibilidades de mostrar sua iniciativa.

Uma alternativa é a utilização de metodologias ativas entrelaçadas com as novas tecnologias, que tem figurado como alternativas para envolver e estimular os alunos no processo de ensino e aprendizagem (LIMA et al., 2018). Em um sentido amplo, toda a aprendizagem é ativa em algum grau, porque exige do aprendiz e dos docentes, formas diferentes de movimentação interna e externa, de motivação, seleção, interpretação, comparação, avaliação, aplicação (MORRAN, 2015). Fatos vem sendo corroborados por teóricos desde a década de 50, como Dewey (1959), Freire (2009), Rogers (1973), Novack (1999), entre outros, que enfatizam, há muito tempo, a importância de superar a educação bancária, tradicional e focar a aprendizagem no aluno, envolvendo-o, motivando-o e dialogando com ele.

A metodologia ativa surgiu a partir de uma série de pesquisas no campo educacional e da pedagogia, que identificaram que uma pessoa aprende melhor quando interage com outras pessoas, com seu objeto de aprendizagem, através da linguagem. A constatação de que a metodologia tradicional já não estava sendo suficiente para o aprendizado partiu da demanda do mercado e dos pais que também demandavam por uma formação diferente.

Neste aspecto, a preocupação com o ensino de geografia no Brasil fica evidenciada ao longo da história, quando se analisa a proposta da Geografia tradicional e da Geografia renovada, na qual a primeira apresentou um limite devido ao tradicionalismo, reflexo do golpe militar, principalmente entre os anos de 1960 e 1970

Esta preocupação transcorreu pelo exercício do ensino em sala e às consequentes abordagens metodológicas para o desenvolvimento e facilitação do aprendizado. Conforme Cavalcanti (2002), o movimento de renovação da Geografia foi marcado pela disputa do poderio de dois núcleos fundamentais, um ligado à uma Geografia considerada "tradicional", que se mantinha tal como havia se estruturado nas primeiras décadas do século XX e, outro, que representava uma Geografia nova, especificamente em uma abordagem crítica (geografia crítica), que buscava suplantar a tradicional, que se autoproclamava "crítica" 
(CAVALCANTI, 1998).

As exigências de formação e capacitação dos alunos para o século XXI têm provocado revisão e transformação das atividades pedagógicas em diferentes níveis. Segundo Candau (2000), por exemplo: A escola precisa ser espaço de formação de pessoas capazes de serem sujeitos de suas vidas, conscientes de suas opções, valores e projetos de referência e atores sociais comprometidos com um projeto de sociedade e humanidade.

Com isso, este tem como objetivo o levantamento de aplicativos livres disponíveis em lojas virtuais (Aplle Store e Play Store) e sites, bem como a avaliação dos principais entraves nos seus usos no processo de ensino e aprendizagem da Geografia.

\section{REVISÃO TEÓRICA}

\section{Metodologias Ativas}

Interessa-se neste artigo especificamente instrumentos e estratégias de avaliação alinhados com metodologias ativas de ensino unidas a novas tecnologias focadas na utilização dos smartfones. Todavia, embora a expressão venha sendo utilizada com ênfase nos últimos anos, a prática 'e mesmo a teoria' não são novas, no que se refere às metodologias ativas. Paulo Freire, por exemplo, embora não utilizasse a expressão, já concebia o docente e o discente como seres ativos no processo de ensino-aprendizagem.

A concepção de atividade também estava presente em Ausubel (1963), inserida no conceito da aprendizagem significativa, um mecanismo humano para adquirir e armazenar a vasta quantidade de ideias e informações representadas em qualquer campo de conhecimento, isto é, quando o significado lógico dos signos de aprendizagem se transforma em significado cognitivo para o sujeito.

Dewey (1959), por sua vez, embora não mencionando a expressão "metodologia ativa", afirmava que o conhecimento se iniciava por um problema e se encerrava com sua resolução, ou seja, seria um processo indagativo, reflexivo e ordenado de ideias. Afinal, todo o ato de pensar seria original, e consequentemente favoreceria a descoberta, possibilitando assim a criação do prazer da produtividade intelectual, para além do simples armazenamento de informações transmitidas por terceiros. Nesse sentido, faziam-se necessárias condições que estimulassem o pensamento para que o aprendizado se desenvolvesse.

Portanto, uma vez que o docente decida se utilizar das metodologias ativas e considere a avaliação como processo para a ação-reflexão-ação de seu trabalho e do aprendizado de seu aluno, faz-se necessário rever os instrumentos da/para a avaliação.

\section{Smartfones}

O smartfone, cujo termo de origem inglesa significa 'telefone inteligente', é um telefone celular com tecnologias avançadas, o que inclui programas e um sistema operacional, equivalente aos computadores. Esses aparelhos possibilitam a qualquer pessoa, com conhecimentos específicos, desenvolver programas para eles, os chamados aplicativos, e existem dos mais variados tipos e para os mais variados objetivos. 0 
smartfone possui características de computador, como hardware e software, pois são capazes de conectar redes de dados para acesso à internet, sincronizar dados como um computador, além da agenda de contatos.

Existem diversos sistemas operacionais para smartfones, tais como Black Berry, iOS, Windows, Android, entre outros, podendo ser das mais diversas marcas e modelos. Os smartfones possibilitam que as pessoas, principalmente jovens, acessem suas informações pessoais e a rede com muito mais velocidade e em aparelhos menores, mas com funções parecidas com as de um computador.

\section{METODOLOGIA}

Iniciaremos aqui a definição e discussão da metodologia que direcionou esta pesquisa e guiou a forma como o objeto de estudo foi avaliado. A figura 1 ilustra as etapas para o desenvolvimento deste artigo, tendo início com a busca dos principais aplicativos que são utilizados pelos professores, em escolas de ensino médio da rede públicas e particulares, na área metropolitana de Santarém, Estado do Pará, Brasil, onde foram aplicados 10 (dez) questionários semiestruturados, em dez escolas diferentes. Para complementar este levantamento foi realizada também pesquisa nos sistemas de buscas na internet, procurando relatos de professores sobre quais aplicativos eram mais utilizados.

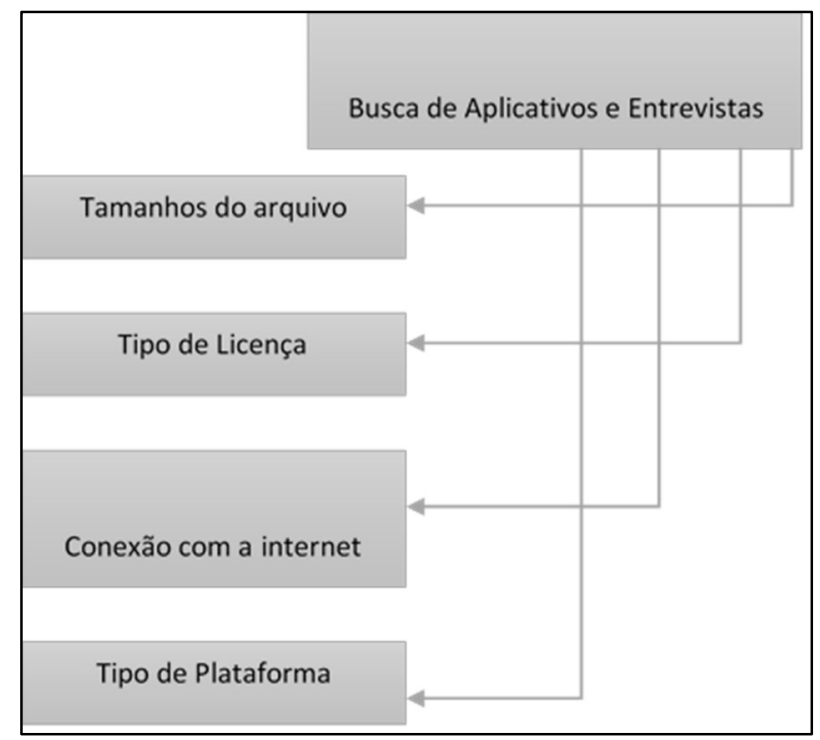

Figura 1: Fluxograma do processo metodológico.

\section{Tamanho dos Arquivos}

Um problema comum nos últimos dez anos, após a popularização dos computadores e dos smartfones, após todo o mundo passar a utilizar mais estes equipamentos, é que os aplicativos mais utilizados no mundo não param de dilatar, sendo necessário cada vez mais memória no equipamento. Claro que as tecnologias tentam se adaptar, cada vez mais, ao crescimento dando mais suporte aos espaços de armazenamentos, no entanto, os modelos recentes não têm sido suficientes para suprir esta necessidade, ou seja, é uma tendência que parece sem volta. Este também é um entrave no que se refere a utilização de metodologias ativas em sala de aula, principalmente quando requerem que os alunos tenham que instalar determinados aplicativos em seus celulares. 


\section{Quanto a distribuição e comercialização}

A distribuição e comercialização de um software é tema de muitas discussões, já que existem opiniões divergentes sobre como isso deveria ser feito. De um lado, os defensores do chamado software livre, que pode ser reproduzido e modificado livremente; de outro, os que acreditam nos softwares proprietários, cujo uso só é permitido com a aquisição de uma licença. Essa divergência gira em torno de direitos autorais e intelectuais, e do custo para o desenvolvimento do software incluindo a remuneração dos profissionais da área de desenvolvimento (gerentes de projeto, analistas de sistemas e programadores (MOLINARI, 2007).

\section{Software livre}

Para a FSF - significado da sigla, 'software livre é uma questão de liberdade, não de preço. Para entender o conceito, você deve pensar em liberdade de expressão, não em cerveja grátis'. O ponto central do software livre se baseia na ideia de garantir liberdade para que usuários executem, copiem, distribuam, estudem, aperfeiçoem e modifiquem um software, levando em consideração suas características, expectativas e necessidades. Tudo isso contribui para uma visão construtivista e evolucionária em que diversas pessoas podem trabalhar para torná-lo melhor.

A FSF menciona que, para que um software seja considerado livre, ele deve necessariamente seguir os quatro princípios da liberdade. São eles: a) Liberdade n.0: o usuário deve ter a liberdade de executar o programa, para qualquer propósito; b) Liberdade n.1: o usuário deve ter a liberdade de estudar e adaptar o programa às suas necessidades. O acesso ao código fonte é um pré-requisito para essa liberdade; c) Liberdade n.2: o usuário deve ter a liberdade de redistribuir cópias de modo que possa ajudar ao próximo; d) Liberdade n.3: o usuário deve ter a liberdade de aperfeiçoar o programa e disponibilizar seus aperfeiçoamentos, de modo a beneficiar toda a comunidade. O acesso ao código fonte é um pré-requisito para essa liberdade.

\section{Open Source}

Também conhecido como código aberto, esse tipo de software também leva em consideração os preceitos de liberdade descritos para o software livre. Sua defesa, porém, a cargo da Open Source Iniciative (OSI), foca mais em questões técnicas (até a eventual superioridade em relação aos softwares proprietários de código fechado) e não tanto em questões éticas ou morais como as que a FSF prega quando se refere ao software livre.

No open source, o código fonte é acessível e há permissão para que seja modificado e redistribuído, mantendo os princípios de liberdade do software original. Isso auxilia na prevenção de sua utilização com fins comerciais, estando sujeito aos termos da licença GPL - significado da sigla. Alguns exemplos de softwares open source são o kernel do sistema operacional Linux e o projeto Web Apache TomCat, que podem ser alterados e redistribuídos. 


\section{Livre comercial}

O software livre comercial ainda preserva a característica de código fonte aberto, mas não exclui a possibilidade de receber um tratamento comercial (distribuição mediante pagamento). No entanto, uma vez que é regido pelas regras da GPL, que exige a sua distribuição sem ônus para o usuário, o software chamado livre comercial encontra outra maneira de ser comercializado. Na maior parte das vezes, trabalha-se com a ideia de agregar valor, diferenciando-o da versão open source. Entre esses recursos, está o empacotamento e a venda com outros softwares integrados, ou a venda de um hardware que se comporta melhor por ser compatível com o software. A distribuição dos softwares Linux RedHat e o Mandrake são alguns exemplos.

O mercado também é fértil em softwares gratuitos, mas nenhum deles abre o código fonte. Só por essa característica, fica claro que software gratuito não é software livre. A impossibilidade do usuário de modificar e redistribuir o software faz com que ele fique indiretamente preso à empresa que o desenvolveu. As versões de software gratuito são duas: a) Versões freeware: podem ser utilizados sem limite de tempo, copiados e distribuídos livremente, mas não podem ser alterados porque o código fonte não é aberto. Exemplo: Java Sun, Internet Explorer; b) Versões hardware: são gratuitos, porém utilizam publicidade, como banners ou links de patrocinadores, que custeiam o desenvolvimento e manutenção em troca de marketing. Como o freeware, podem ser copiados e distribuídos livremente, mas sem modificações (pela falta do código fonte). Exemplo: Adobe Acrobat.

\section{Conexão de internet}

Prega-se que para falar a língua de crianças e adolescentes os educadores precisam saber explorar o potencial dos novos recursos tecnológicos. Segundo Varella (2017) "Em escolas públicas brasileiras, 81\% delas têm laboratórios de informática, mas somente 59\% deles são usados". Entretanto, já na década de 90, a internet já estava explodindo como a mídia mais promissora desde a implantação da televisão, sendo a mídia mais aberta, descentralizada, e, por isso mesmo, mais ameaçadora para os grupos políticos e econômicos hegemônicos. Aumenta o número de pessoas ou grupos que criam na Internet suas próprias revistas, emissoras de rádio ou de televisão, sem pedir licença ao Estado ou ter vínculo com setores econômicos tradicionais. Cada um pode "dizer nela o que quiser", conversar com quem desejar, oferecer os serviços que considerar convenientes. Como resultado, começamos a assistir a tentativas de controlá-la de forma clara ou sutil (MORRAN, 1997). As redes atraem os estudantes. Eles gostam de navegar na rede, de descobrir endereços novos, de divulgar suas descobertas, de se comunicar com outros colegas. Mas também podem perder-se entre tantas conexões possíveis, tendo dificuldade em escolher o que é significativo, em fazer relações, em questionar afirmações problemáticas.

\section{RESULTADOS E DISCUSSÃO}

\section{Levantamento de Aplicativos}

O quadro 1 mostra uma breve descrição e o levantamento dos aplicativos disponíveis, encontrados 
nas buscas e pesquisas na web, bem como, os utilizados em redes de escolas públicas da cidade de

Santarém/PA. Em virtude das buscas online revelam uma grande infinidade de aplicativos, optou-se por trabalhar com os que surgiam mais frequentemente nos sites de busca.

Quadro 1: Levantamento e descrição dos aplicativos.

\begin{tabular}{|c|c|}
\hline $\begin{array}{l}\text { Nome do } \\
\text { Software }\end{array}$ & Descrição \\
\hline Kahooh & $\begin{array}{l}\text { Este é um Quizzes e games, que é uma ótima estratégia para captar a atenção dos jovens. Desta forma, } \\
\text { a plataforma Kahoot vem se destacando como uma das estratégias de aprendizado mais comuns, sendo bastante eficaz na } \\
\text { sala de aula. } \\
\text { Os professores são como apresentadores de um programa de TV, onde lançam perguntas e os estudantes, que podem ser } \\
\text { divididos em grupos, têm um determinado tempo para responder corretamente. }\end{array}$ \\
\hline Plickes & $\begin{array}{l}\text { É um aplicativo de interação entre educadores e estudantes a partir da criação de quizzes, digitais e em tempo real, permite } \\
\text { uma avaliação através da plataforma, não torna a obrigatoriedade do download do aplicativo para os alunos, simplesmente } \\
\text { requer que o professor possua o app e imprima cartões de realidade aumentada, os quais são distribuídos aos alunos em } \\
\text { sala de aula. }\end{array}$ \\
\hline $\begin{array}{l}\text { Google for } \\
\text { Education }\end{array}$ & $\begin{array}{l}\text { O Google oferece uma infinidade de aplicativos voltada para a educação, popularmente conhecidos como G-Suite for } \\
\text { Education, estas ferramentas são disponibilizadas gratuitamente, tanto para o sistema Android quanto para iOS, e podem } \\
\text { contribuir para auxiliar na transformação da sala de aula, deixando-as mais dinâmica. }\end{array}$ \\
\hline Expeditions & $\begin{array}{l}\text { Uma das diversas ferramentas disponibilizadas no G Suite, que merece destaque, é o Expeditions. Possibilita uma } \\
\text { experiência imersiva de realidade virtual, que pode ser feita tanto pelo celular quanto com a ajuda de óculos 3D. Uma } \\
\text { alternativa é o professor fazer o download de uma determinada expedição - por exemplo, pela Muralha da China, uma } \\
\text { espécie de rede conectada ficará à disposição para ser compartilhada com os alunos, que podem acessar aquele conteúdo } \\
\text { em seus próprios celulares. }\end{array}$ \\
\hline LightBot & $\begin{array}{l}\text { Com o desenvolvimento das novas tecnologias, a programação vem ganhado espaço no currículo escolar, e já é } \\
\text { considerada uma competência importante para a nova geração. O LightBot, é um aplicativo disponível gratuitamente tanto } \\
\text { na para plataformas Android e iOS, que disponibiliza a oportunidade de aprender a codificar através de jogos e puzzles. O } \\
\text { objetivo é desenvolver habilidades de lógica, sequenciação e condicionais, mas sem introduzi-las como conceito, apenas } \\
\text { contando com os aspectos lúdicos dos games. }\end{array}$ \\
\hline ProDeaf & $\begin{array}{l}\text { É um aplicativo com intuito de facilitar a comunicação e a inclusão de deficientes auditivos, o tem a capacidade de traduzir } \\
\text { texto e vozes em português para Libras (Língua Brasileira de Sinais), o processo inverso também ocorre, traduz o sinal em } \\
\text { Libras para texto e voz, este foi desenvolvido por um startup brasileira. }\end{array}$ \\
\hline RECAP & $\begin{array}{l}\text { É uma plataforma interligada em que alunos e professores aprofundam discussões a respeito de questões, usando } \\
\text { ferramentas de multimídia disponibilizadas no aplicativo, permitindo que os pais, também possam acompanhar a } \\
\text { desenvolvimento da aprendizagem de seus filhos. Um entrave nesta plataforma é que exige que os estudantes façam o } \\
\text { download do aplicativo, e nem sempre garante a inclusão de toda a turma. }\end{array}$ \\
\hline $\begin{array}{l}\text { Seterra: Quiz } \\
\text { de Geografia }\end{array}$ & $\begin{array}{l}\text { O app Seterra possui cerca de } 300 \text { quizzes de diferentes mapas que permitem testar seus conhecimentos de Geografia. } \\
\text { Possibilita aprendizagem, como por exemplo distinguir a Tasmânia, na Austrália, da Tanzânia, em África, e a bandeira bleu, } \\
\text { blanc, Rouge, da França, das listas brancas, azuis e vermelhas da Rússia. Cidades, países, capitais, continentes e as grandes } \\
\text { massas de água fazem parte do jogo. Encontre o Kilimanjaro e o Monte McKinley no mundo das montanhas ou descubra } \\
\text { ilhas exóticas em partes distantes do globo aceitando o desafio das ilhas do mundo. }\end{array}$ \\
\hline ActiveTrack & $\begin{array}{l}\text { A app ActiveTrack permite o desenvolvimento de trajetórias ativas, ou seja, percursos que interagem com o utilizador, por } \\
\text { exemplo para roteiros de visitas de estudo ou atividades de exploração. O usuário, ao longo do percurso, tem a função da } \\
\text { proximidade de coordenadas previamente selecionadas, recebe informação e tarefas permitindo interagir com conteúdo } \\
\text { sobre a atividade e o ponto em que se encontra. }\end{array}$ \\
\hline $\begin{array}{l}\text { Stack the } \\
\text { countries }\end{array}$ & $\begin{array}{l}\text { Este aplicativo possibilita a aprendizagem sobre conteúdos de diferentes países do mundo, como suas capitais, as suas } \\
\text { características mais relevantes, a localização geográfica e aspectos culturais. }\end{array}$ \\
\hline Earth Primer & $\begin{array}{l}\text { Mostra o funcionamento do planeta terra através de um jogo, onde possibilita a criação de vulcões, molde de montanhas e } \\
\text { controle as forças da natureza com a ponta dos dedos, ele possibilita por exemplo a criação de uma ilha, ou descobrir como } \\
\text { criar chuva. Earth Primer desafia os aplicativos existentes do géneros, combinando aspectos de livros científicos, } \\
\text { brinquedos, simulações e jogos. É um novo tipo de experiência interativa que combina a qualidade de um livro de Geografia } \\
\text { com um jogo de simulação. }\end{array}$ \\
\hline $\begin{array}{l}\text { Google } \\
\text { Expeditions }\end{array}$ & $\begin{array}{l}\text { É uma ferramenta de realidade virtual que permite conduzir ou participar de viagens virtuais imersivas em todo o mundo. } \\
\text { Aproxime-se de marcos históricos, mergulhe debaixo de água com tubarões e visite o espaço exterior. } \\
\text { Construído para uso em sala de aula e em pequenos grupos, o Google Expeditions permite que o professor desempenhe o } \\
\text { papel 'guia' para conduzir grupos de 'exploradores' na sala de aula através de coleções de imagens em 360 e 3D, } \\
\text { apontando pontos interessantes ao longo do caminho. Ligue os dispositivos dos alunos à mesma rede Wi-Fi- se o Guia } \\
\text { (professor) tiver feito o download de algumas Expedições, não é necessária ligação à Internet para executar o Expedition! A } \\
\text { app funciona com o visor Google Cardboard (http://g.co/cardboard) }\end{array}$ \\
\hline $\begin{array}{l}\text { Geografia } \\
\text { Mundial - }\end{array}$ & $\begin{array}{l}\text { É um jogo de perguntas que irá auxiliar na aprendizagem sobre países, direcionando a temas como mapas, bandeiras, } \\
\text { capitais, população, religião, idioma, moeda, permite ainda perguntas em português, que irá auxiliá-lo a aprender tudo } \\
\text { sobre a Geografia. }\end{array}$ \\
\hline
\end{tabular}

\section{Da origem do levantamento}

O Quadro 2 traz a origem do levantamento dos aplicativos, que foram pesquisados nas escolas públicas e particulares, bem como, na rede de internet web. A maior parte dos aplicativos são resultados de buscas online na web, mesmo aplicativos, como Kahooh e Plickres, que são usados tanto nas redes públicas 
e particulares, são bastante citados na internet.

Isso nos revela pouco uso de metodologias ativas e smartfones no processo de ensino e aprendizagem, mesmo em redes de escolas particulares da região norte do país, como em Santarém, as mais prováveis causas, são as dificuldades com a internet e as próprias condições da educação na região norte, outro agravante é o perfil socioeconômico dos estudantes.

Quadro 2: Da origem do levantamento.

\begin{tabular}{|l|l|l|l|}
\hline \multirow{2}{*}{ Nome do Software } & \multicolumn{2}{c|}{ Origem do Levantamento } \\
\cline { 2 - 4 } & \multicolumn{2}{|c|}{ Rede Publica } & \multicolumn{2}{c|}{ Rede Particular } & Buscas na web \\
\hline Kahooh & Sim & Sim & Sim \\
\hline Plickres & Sim & Sim & Sim \\
\hline Google for Education & Não & Não & Sim \\
\hline xExpeditions & Não & Não & Sim \\
\hline LightBot & Não & Não & Sim \\
\hline ProDeaf & Não & Não & Sim \\
\hline RECAP & Não & Não & Sim \\
\hline Seterra - Quiz de geografia & Não & Não & Sim \\
\hline ActiveTrack & Não & Não & Sim \\
\hline Stack the countries & Não & Não & Sim \\
\hline Earth Primer & Não & Não & Sim \\
\hline Google Expeditions & Não & Sim & Sim \\
\hline Geografia Mundial - Jogo & Não & Sim & Sim \\
\hline
\end{tabular}

\section{Qual Tamanho em Megabytes do APP}

A tabela 1 traz o tamanho dos aplicativos em megabites, para as diferentes plataformas (Android e IOS), bem como, a diferença do tamanho dos aplicativos nas duas plataformas em porcentagem. Os aplicativos para a plataforma Android tem um tamanho com uma média de aproximadamente de 25,65 Mb, já no sistema IOS temos uma média de 64,95Mb cerca de 1,5 maior que o Android. Para um mesmo aplicativo, quando o sistema é o IOS, estes são maiores, ou seja, ocupam um volume de espaço considerável nos smartfones. Este ponto é importante, pois para os alunos que irão baixar um aplicativo, podem considerar um entrave o espaço que vai ocupar nos seus equipamentos, sendo, portanto, este um fator limitante.

Tabela 1: Tamanho em MB dos aplicativos para diferentes sistemas.

\begin{tabular}{|l|l|l|l|}
\hline \multirow{2}{*}{ Nome do Software } & \multicolumn{2}{c|}{ Tamanho (MB) } & \multicolumn{1}{c|}{ Diferença tamanho Androide/IOs em \% } \\
\cline { 2 - 4 } & \multicolumn{2}{|c|}{ Androide } & \multicolumn{1}{c|}{ IOS } \\
\hline Kahooh & 24 & 36,3 & $151,25 \%$ \\
\hline Plickes & 16 & 28,9 & $180,63 \%$ \\
\hline Google for Education & 11 & 131,4 & $1194,55 \%$ \\
\hline Expeditions & 37 & 89,1 & $240,81 \%$ \\
\hline LightBot & 20 & 44 & $220,00 \%$ \\
\hline ProDeaf & 19 & 53,9 & $283,68 \%$ \\
\hline RECAP & 35 & - & \\
\hline Seterra - quiz de geografia & 59 & 146,7 & $248,64 \%$ \\
\hline ActiveTrack & 1 & - & $0,00 \%$ \\
\hline Stack the countries & 33 & 174,2 & $527,88 \%$ \\
\hline Earth Primer & 15 & - & \\
\hline Google Expeditions & 37 & 89,1 & $240,81 \%$ \\
\hline Geografia Mundial - Jogo & 26 & 50,8 & $195,38 \%$ \\
\hline TOTAL & $\mathbf{3 3 3 , 0 0}$ & $\mathbf{8 4 4 , 4 0}$ & \\
\hline MÉDIA & $\mathbf{2 5 , 6 2}$ & $\mathbf{6 4 , 9 5}$ & \\
\hline
\end{tabular}

\section{Apresenta Multiplataformas}

O Quadro 3 ilustra em três diferentes plataformas Android, IOS e em linguagem $h t t p$, neste é possível 
de maneira geral observar que grande parte dos aplicativos investigados estão disponíveis em diferentes plataformas.

Quadro 3: Aplicativos em diferentes plataformas.

\begin{tabular}{|l|l|l|l|}
\hline \multicolumn{1}{|c|}{ Nome do Software } & \multicolumn{1}{c|}{ Androide } & IOS \\
\hline Kahooh & Sim & Sim & Sim \\
\hline Plickres & Sim & Sim & \\
\hline Google for Education & Sim & Sim & \\
\hline Expeditions & Sim & Sim & \\
\hline LightBot & Sim & Sim & Não \\
\hline ProDeaf & Sim & Não & Não \\
\hline RECAP & Sim & Não & \\
\hline Seterra - quiz de geografia & Sim & Sim & \\
\hline ActiveTrack & Sim & Não & \\
\hline Stack the countries & Sim & Sim & não \\
\hline Earth Primer & Sim & Sim & \\
\hline Google Expeditions & Sim & Sim & \\
\hline Geografia Mundial - Jogo & Sim & \\
\hline
\end{tabular}

\section{Tipo de Licença}

O Quadro 4 ilustra os tipos de licença dos aplicativos, nestes é possível observar que $100 \%$ dos aplicativos avaliados possuem uma versão licença free, entretanto apresentam limitações em suas funções, e ainda trazem propagandas no desenvolvimento das atividades.

Quadro 4: Tipos de licenças nos aplicativos avaliados.

\begin{tabular}{|c|c|c|c|c|c|}
\hline Nome do Software & Licença Free & Open Soucer & Livre Comercial & Propagandas & Versão Proprietário \\
\hline Kahooh & Sim & & & Não & Sim \\
\hline Plickres & Sim & & & Não & Sim \\
\hline Google for Education & Sim & & & Sim & Sim \\
\hline Expeditions & Sim & & & Sim & Não \\
\hline LightBot & Sim & & & Sim & Não \\
\hline ProDeaf & Sim & & & Sim & Não \\
\hline RECAP & Sim & & & Sim & Não \\
\hline Seterra - quiz de geografia & Sim & & & Sim & Não \\
\hline ActiveTrack & Sim & & & Sim & Não \\
\hline Stack the countries & Sim & & & Sim & Não \\
\hline Earth Primer & Sim & & & Sim & Não \\
\hline Google Expeditions & Sim & & & Não & Não \\
\hline Geografia Mundial - Jogo & $\operatorname{Sim}$ & & & Não & Sim \\
\hline
\end{tabular}

\section{Requer conexão com a internet}

O Quadro 5 apresenta os aplicativos e sua relação com a internet, ou seja, se para seu uso é necessário estar conectado à internet. Neste aspecto fica clara a dependência a rede mundial de computadores no processo de aprendizagem, pois os 13 aplicativos avaliados, somente um (Plickres) não requer que os alunos possuam internet. Este fato sem dúvida se torna um entrave na aplicação destes métodos em sala de aula, principalmente em regiões brasileiras, onde a disponibilidade de internet é limitada, no que se refere ao acesso ou fluxo de dados, e a disponibilidade e acessibilidade do serviço.

Quadro 5: Aplicativos e sua relação com a internet.

\begin{tabular}{|l|l|l|}
\hline \multicolumn{1}{|c|}{ Nome do Software } & \multicolumn{1}{c|}{ Online } & Off-line \\
\hline Kahooh & Sim & \\
\hline Plickes & Sim & Sim \\
\hline Google for Education & Sim & \\
\hline Expeditions & Sim & \\
\hline LightBot & Sim & \\
\hline ProDeaf & Sim & \\
\hline RECAP & Sim & \\
\hline
\end{tabular}




\begin{tabular}{|l|l|l|}
\hline Seterra - quiz de geografia & Sim \\
\hline ActiveTrack & Sim \\
\hline Stack the countries & Sim & \\
\hline Earth Primer & Sim & \\
\hline Google Expeditions & Sim & \\
\hline Geografia Mundial - Jogo & Sim & \\
\hline
\end{tabular}

\section{CONCLUSÕES}

A introdução de novas tecnologias na educação vem sendo amplamente debatida, entretanto ainda pouco conclusiva, mesmo se tornando rotineiro nas escolas diariamente, a tecnologia ainda impõe inúmeros desafios e uma diversidade de entraves. Isso se fortalece uma vez que alguns educadores temem que o uso da internet e dos softwares prejudiquem o processo de aprendizagem, outros, no entanto, percebem o potencial da tecnologia em agregar metodologias ativas no processo educacional e a utilizam em seus planejamentos.

Neste contexto, gestores, principalmente em escolas particulares, anunciam em suas campanhas de matrículas as novas tecnologias. $\mathrm{O}$ uso destas tecnologias que, em primeiro momento pode parecer mais um complicador na difícil tarefa de gestão do ambiente escolar, acaba se mostrando como solução simplificadora na medida em que pequenas ações vão se somando e produzindo uma escola mais dinâmica e com ensino de melhor qualidade.

\section{REFERÊNCIAS}

AUSUBEL, D. P.. The psychology of meaningful verbal learning. New York: Grune and Stratton, 1963.

CANDAU, V. M.. Reinventar a escola. Petrópolis: Vozes, 2000.

CAVALCANTI, L. S.. Geografia e práticas de ensino. Goiânia: Alternativa, 2002.

CAVALCANTI, L. S.. Geografia, escola e construção de conhecimentos. 4 ed. Campinas: Papirus, 1998.

DEWEY, J.. Vida e Educação. São Paulo: Nacional, 1959.

FREIRE, P.. Pedagogia da autonomia: saberes necessários à prática educativa. 33 ed. São Paulo: Paz e Terra, 2009.

GOIS, D. V.; BEZERRA, J. B.. Metodologias ativas no ensino de geografia na educação básica. 2018.

LIMA, V. Y.; SILVA, P. V. N.; MACÊDO, V. F.. O acesso aos recursos tecnológicos ao ensino superior: $O$ que dizem os alunos do Curso de Matemática da UFPI Campus de Picos. 2018.

NOVACK, J. D.; GOWIN, D. B.. Aprender a aprender. 2 ed. Lisboa: Plátano, 1999.

ROGERS, C.. Liberdade para aprender. Belo Horizonte: Interlivros, 1973.

MOLINARI, L.. Gerência de configuração: técnicas e práticas no desenvolvimento do software. Florianópolis: Visual Books, 2007.

MORAN, J. M.. Mudando a educação com metodologias ativas. In: Convergências Midiáticas, Educação e Cidadania: aproximações jovens. São Paulo: USP, 2015.

VARELLA, P. G.. Aprendizagem Colaborativa Em Ambientes Virtuais De Aprendizagem: A Experiência Inédita Da PUC-PR. Revista Diálogo Educacional, Curitiba, v.3, n.6, p.11-27, 2002.

A CBPC - Companhia Brasileira de Produção Científica (CNPJ: 11.221.422/0001-03) detém os direitos materiais desta publicação. Os direitos referem-se à publicação do trabalho em qualquer parte do mundo, incluindo os direitos às renovações, expansões e disseminações da contribuição, bem como outros direitos subsidiários. Todos os trabalhos publicados eletronicamente poderão posteriormente ser publicados em coletâneas impressas sob coordenação da Sustenere Publishing, da Companhia Brasileira de Produção Científica e seus parceiros autorizados. Os (as) autores (as) preservam os direitos autorais, mas não têm permissão para a publicação da contribuição em outro meio, impresso ou digital, em português ou em tradução. 